Conclusion* In neoplasms where the initial symptoms are mild, such as endometrial cancer, the number of cases has been reduced, which may be due to the lack of consultation by patients. Regarding ovarian cancer, we have not seen a decrease in cases, although we have seen an increase in the stage. It is expected that the consequences of the COVID pandemic will continue to be felt in the future with an increase in the advanced stages of these neoplasms.

\section{ACS NSQIP - PERSONALISED RISK PREDICTION TOOL FOR POSTOPERATIVE COMPLICATIONS IN GYNAEONCOLOGY SURGERY?}

${ }^{1} \mathrm{~L}$ Sevinyan*, ${ }^{2} \mathrm{~S}$ Jones, ${ }^{3} \mathrm{~J}$ Horne, ${ }^{3} \mathrm{R}$ Bharathan, ${ }^{1} \mathrm{~A}$ Tailor, ${ }^{1} \mathrm{~S}$ Butler-Manuel, ${ }^{4} \mathrm{P}$ Williams, ${ }^{1,5}$ T Madhuri. ${ }^{1}$ Royal Surrey County Hospital, Gynaeoncology, Guildford, UK; ${ }^{2}$ Cardiff University, UK; ${ }^{3}$ University Hospitals of Leicester, UK; ${ }^{4}$ University Of Surrey, UK; ${ }^{5}$ University Of Brighton, UK

\subsection{6/ijgc-2021-ESG0.315}

Introduction/Background* Despite the informed consent process, patients' understanding of potential post-operative complications is often limited, making it difficult to call the decision an informed one, so estimating the risk of postoperative complications is important for shared decision making and to help multidisciplinary teams plan postoperative care. Increased incidence of gynaecological cancers and operations, especially technically challenging minimally invasive surgery (MIS) in older, obese and patients with multiple comorbidities, requires accurate prediction of the likelihood of mortality and morbidity and patient involvement in joint decision making about the management. ACS-NSQIP (American College of Surgeons National Surgical Quality Improvement Program) risk calculator is a validated web-based tool based on 21 preoperative risk factors to predict 8 post-operative outcomes. The objective of our study was to explore the validity of ACS NSQIP in gynaeoncology for perioperative prediction of postoperative complications.

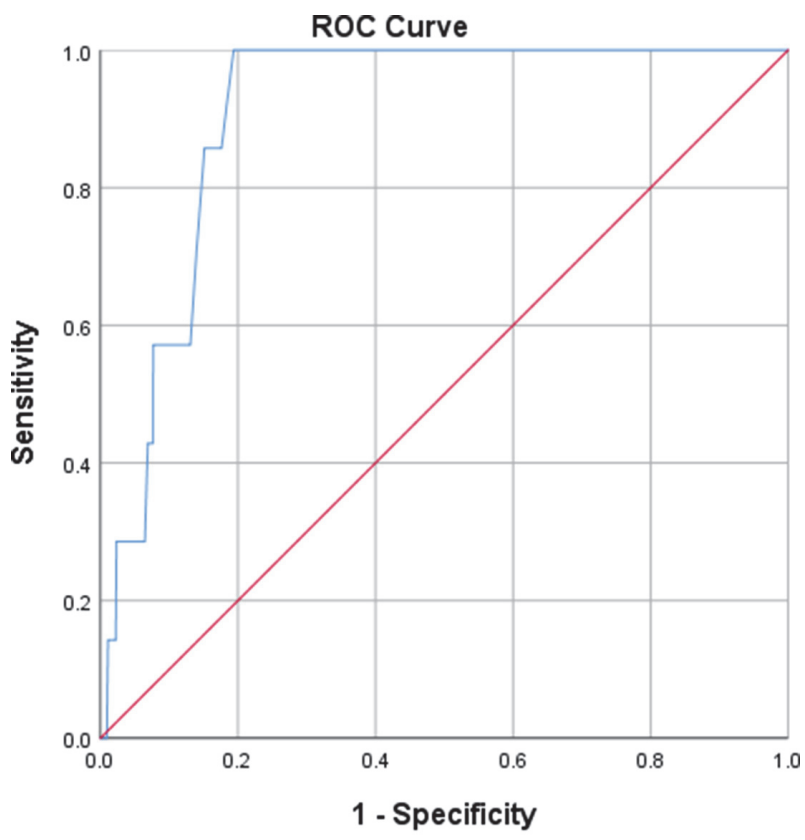

Diagonal segments are produced by ties
Methodology A retrospective multicentre cohort study evaluated 1552 patients who underwent surgery at a tertiary oncology centre. Data collection undertaken through dedicated database. Data collated on 764 patients undergoing robotic, 248 laparoscopic and 540 open surgery for suspected or confirmed gynaecological malignancy. All missing data collated from patient notes. Following data lock with the actual postop event/complication that occurred in this retrospective cohort, ACS NSQIP used to count predictive scores for each patient. Data analysis evaluating ACS-NSQIP validity and relevance in gynaecological oncology patients and its ability to predict postoperative complications performed.

Result(s)* ACS-NSQIP was found to best predict mortality (AUC - 0.908), it was most accurate for prediction of complications as follows: discharge to rehabilitation (AUC-0.875), cardiac complications (AUC-0.854), sepsis (AUC-0.795), pneumonia (AUC-0.764), return to theatre (AUC-0.696), surgical site infection (AUC-0.686), VTE (AUC-0.676), readmission (AUC-0.669), renal failure (AUC-0.601). Poor result in the prediction of UTI (AUC-0.543) was noted.

Conclusion* ACS-NSQIP risk calculator appears to predict major complications and post-operative mortality making it useful as an informed consent tool. Preliminary data suggests that further validation is required to fully evaluate if the risk scores may be used to inform patients pre-operatively of their risk of complications and is currently being undertaken.

\section{APPLICABILITY OF PRE-OPERATIVE PATIENT REPORTED DUKE ACTIVITY SCALE INDEX IN PREDICTION OF POSTOPERATIVE COMPLICATIONS IN GYNAECOLOGICAL ONCOLOGY}

${ }^{1}$ L Sevinyan*, 'A Tailor, ${ }^{2} \mathrm{P}$ Prabhu, ${ }^{3} \mathrm{P}$ Williams, ${ }^{1,4} \mathrm{~T}$ Madhuri. ${ }^{1}$ Royal Surrey County Hospital, Gynaeoncology, UK; ${ }^{2}$ Royal Surrey County Hospital, Anaesthetics, UK; ${ }^{3}$ University Of Surrey, UK; ${ }^{4}$ University Of Brighton, UK

\subsection{6/ijgc-2021-ESGO.316}

Introduction/Background* Increase in the incidence of gynaecological cancers has resulted in increased operative procedures, specifically in patients with multiple comorbidities including obesity and frailty. This is often associated with prolonged admission and higher rates of postoperative mortality and morbidity and presents a challenge with an unmet need for an accurate, personalised risk prediction. Duke Activity Scale Index (DASI) is a 12 item scale in the form of selfreported questionnaire based around commonly performed activities of daily living. Currently, DASI is used to evaluate patients with cardiovascular diseases, however there is growing interest in utilising it in preoperative setting in different specialities. This study investigates the accuracy of DASI in preoperative prediction of postoperative outcomes in gynaeoncology.

Methodology A retrospective cohort study of 486 patients who had undergone an operative treatment at a tertiary oncology centre. Data collection undertaken through dedicated gynaeoncology database and missing data collected through patients' records. All patients had completed the DASI questionnaire prior to their consultation. Actual postoperative 30 day complications and the length of stay also recorded. DASI was then compared with the occurrence of postoperative complications.

Result(s)* 242 patients had a Da Vinci robot-assisted procedure, 44 - laparoscopic and 200 - open surgery. 133/486 were 


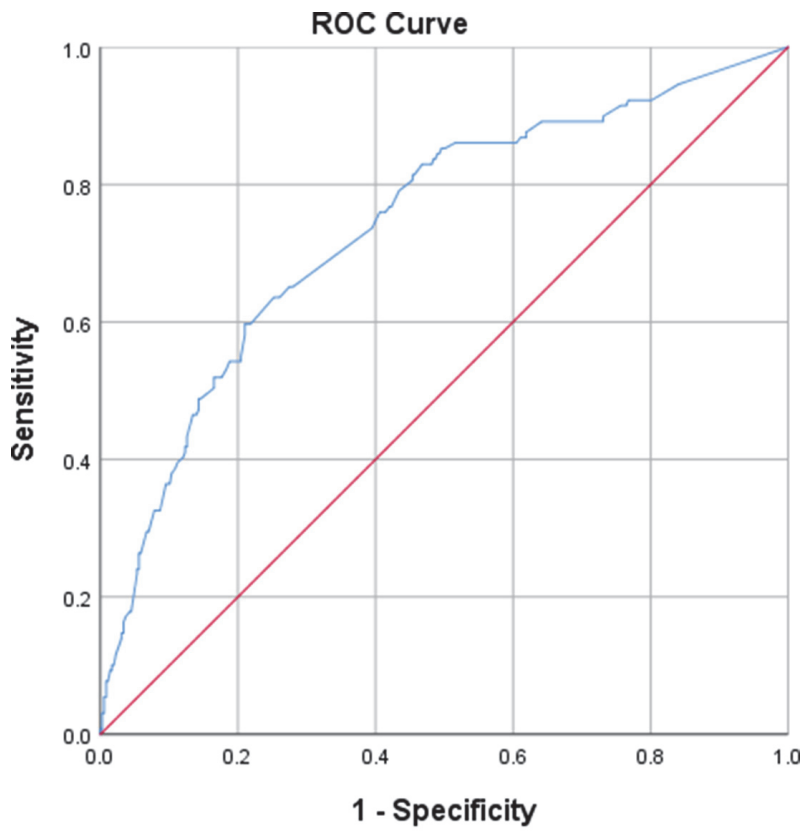

Diagonal segments are produced by ties.

\section{Abstract 726 Figure 1}

classified as having any type of complications within 30 days of the operation. Our results have shown that the higher DASI score the less likely patients were to have postoperative complications. This result was statistically significant with odds ratio of 0.973 and confidence interval between 0.961 and 0.986. We were also able to demonstrate that for every 10 points further up the DASI score a patient was 0.761 times less likely to have a postoperative complication. Hence general morbidity prediction of DASI score has been found to statistically significantly predict postoperative complications (AUC0.740).

Conclusion* Our study has shown that DASI self-reported score is a useful predictive tool of perioperative estimation of postoperative complications in the gynaeoncology setting. Further analysis with a larger sample size and a multicentre prospective study is currently underway to validate the findings.

\section{EUROPEAN ENHANCED RECOVERY AFTER SURGERY (ERAS) GYNECOLOGICAL ONCOLOGY SURVEY: CURRENT STATE OF PERIOPERATIVE PRACTICE}

${ }^{1}$ NR Gómez-Hidalgo*, ${ }^{2} \mathrm{~A}$ Pletnev, ${ }^{3} \mathrm{Z}$ Razumova, ${ }^{4} \mathrm{~N}$ Bizzarri, ${ }^{5}$ Selcuk, ${ }^{6} \mathrm{C}$ Theofanakis, ${ }^{7} \mathrm{~K}$ Zalewski, ${ }^{8} \mathrm{~T}$ Nikolova, ${ }^{9} \mathrm{M}$ Lanner, ${ }^{10} \mathrm{~J}$ Kacperczyk-Bartnik, ${ }^{1} \mathrm{U}$ Acosta, ${ }^{1} \mathrm{~PB}$ Asuncion, ${ }^{1} \mathrm{~A}$ Gil-Moreno, ${ }^{11} \mathrm{G}$ Nelson, ${ }^{12} \mathrm{C}$ Fotopoulou, ${ }^{1} \mathrm{JL}$ Sánchez Iglesias. ${ }^{1}$ Hospital Universitari Vall d'Hebron, Gynecology Oncology, Barcelona, Spain; ${ }^{2}$ Alexandrov National Cancer Centre of Belarus, Gynecology Oncology, Belarus; ${ }^{3}$ Karolinska Institute, Gynecology Oncology, Sweden; ${ }^{4}$ Fondazione Policlinico Universitario A. Gemelli, Roma, Italy; ${ }^{5}$ Maternity Hospital, Ankara City Hospital, Ankara, Turkey; ${ }^{6}$ Alexandra General Hospital, Athina, Greece; ${ }^{7}$ Świętokrzyskie Cancer Centre, Kielce, Poland; ${ }^{8}$ Heidelberg University, Heidelberg, Germany; ${ }^{9}$ Cardinal Schwarzenberg Hospital, Schwarzach im Pongau, Austria; ${ }^{10}$ Medical University of Warsaw, Warszawa, Poland; ${ }^{11}$ Cumming School of Medicine U C, Calgary, Canada; ${ }^{12}$ Imperial College Healthcare NHS Trust, UK

\subsection{6/ijgc-2021-ESG0.317}

Introduction/Background* Enhanced Recovery After Surgery (ERAS) reduces complication rates after surgery, decreases length of hospital stay (LOS) and reduces costs. A correct

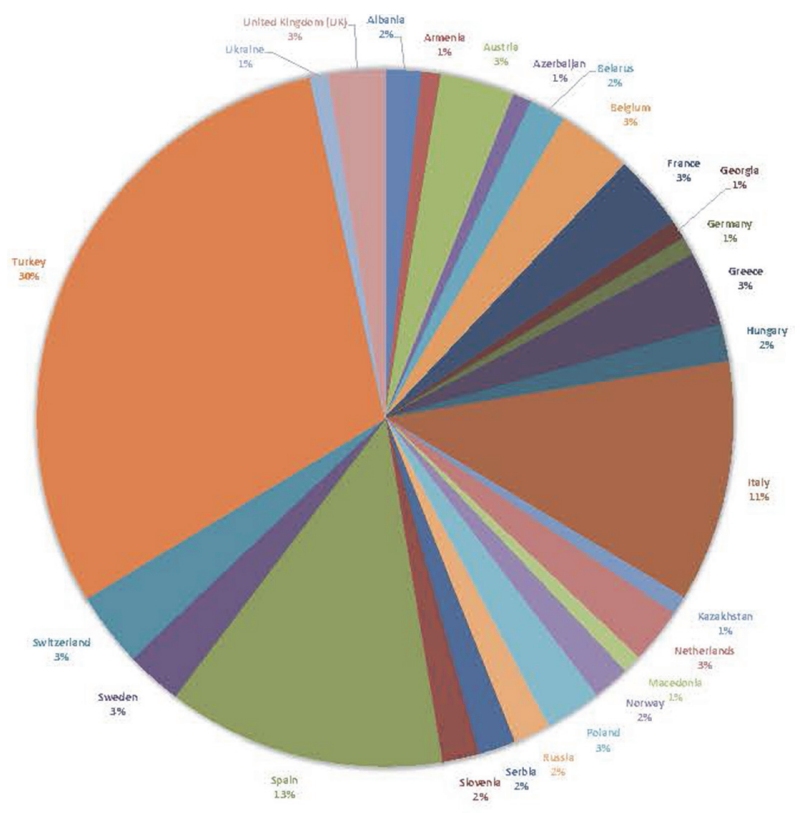

Abstract 757 Figure 1 Participating European Centres
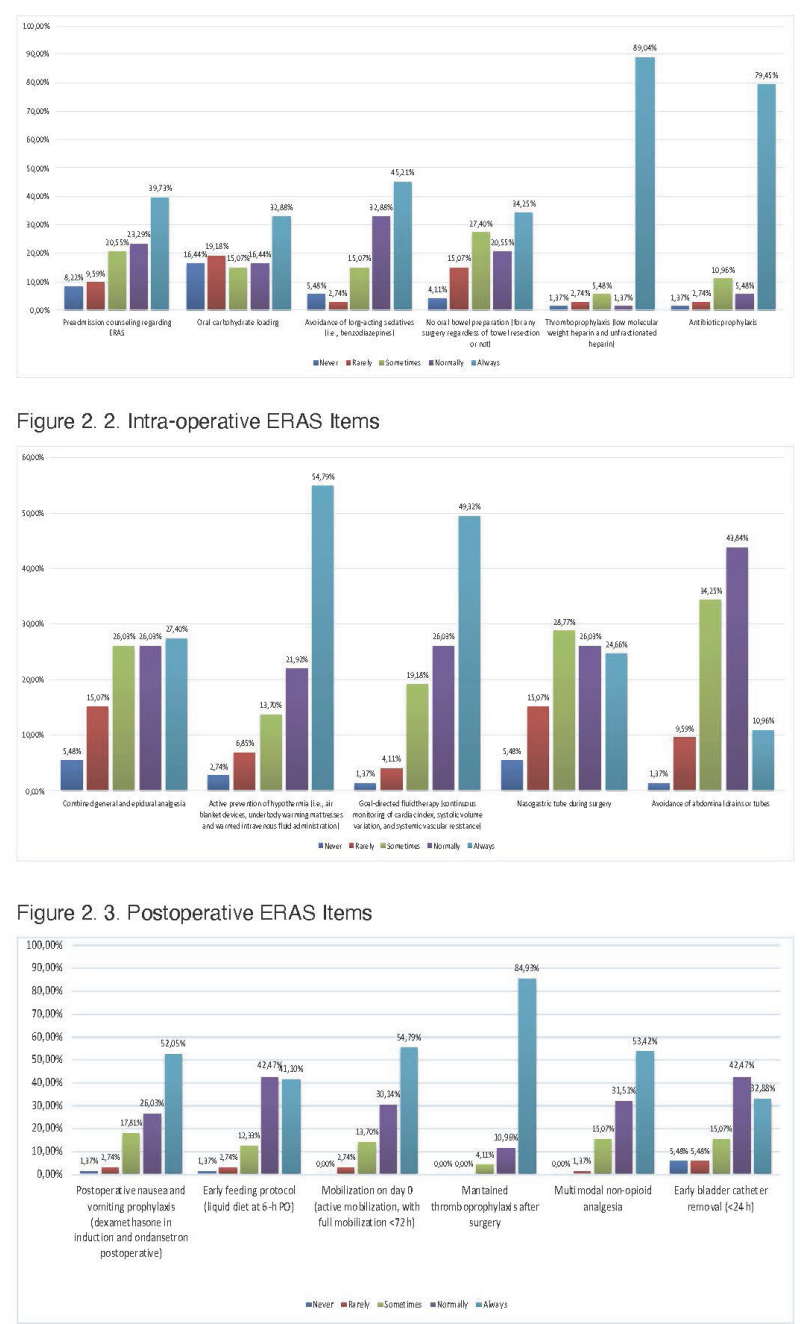

Abstract 757 Figure 2 Centers Practicing ERAS Items 